\title{
How well can inaccurate sensors quantify and improve the performance of a fleet of on-site wastewater treatment plants?
}

Schneider, Mariane Yvonnea, b, e; Harada, Hidenoric; Villez, Kris ${ }^{d}$; Maurer, Maxa, b

a Eawag, Swiss Federal Institute of Aquatic Science and Technology, 8600 Dübendorf, Switzerland.

b Institute of Civil, Environmental and Geomatic Engineering, ETH Zürich, 8093 Zurich, Switzerland.

c Graduate School of Asian and African Area Studies, Kyoto University, Yoshida-Shimoadachi, Sakyo, Kyoto 6068501, Japan

d Oak Ridge National Laboratory, Oak Ridge, TN 37831, USA.

e Next Generation Artificial Intelligence Research Center \& School of Information Science and Technology, The University of Tokyo, 7-3-1 Hongo, Bunkyo-ku, Tokyo 113-8656, Japan.

*Contact Address: Max Maurer Eawag, Swiss Federal Institute of Aquatic Science and Technology. Urban Water Management Überlandstrasse 133, 8600 Dübendorf, Switzerland.

E-mail: max.maurer@eawag.ch (M. Maurer)

Copyright notice:

This manuscript has been authored by UT-Battelle, LLC under Contract No. DE-AC05-00OR22725 with the U.S. Department of Energy. The United States Government retains and the publisher, by accepting the article for publication, acknowledges that the United States Government retains a non-exclusive, paid-up, irrevocable, world-wide license to publish or reproduce the published form of this manuscript, or allow others to do so, for United States Government purposes. The Department of Energy will provide public access to these results of federally sponsored research in accordance with the DOE Public Access Plan (http://energy.gov/downloads/doe-public-access-plan). 


\begin{abstract}
On-site wastewater treatment plants (OSTs) are widely seen as a stopgap solution, mainly because of a lack of monitoring and the resulting unreliable treatment performance. To address this concern, low maintenance, but inaccurate soft sensors are emerging. However, the impact of this inaccuracy on the treatment performance of entire fleets of OSTs has not been quantified. We develop a stochastic model to estimate these performances.

In the modelled case soft sensors with a $70 \%$ accuracy improve the treatment performance from $66 \%$ (percentage of time functional) to $98 \%$. Soft sensors optimised for specificity (true negative rate) improve the system performance, while such optimised for sensitivity (true positive rate) quantify the treatment performance more accurately. Based on this new insight we suggest to build two soft sensors with the same data input in practical settings: one soft sensor geared towards high specificity, for maintenance scheduling, and one geared towards high sensitivity, for fleet performance quantification. The findings suggest that inaccurate sensors in combination with an appropriate alarm management have the potential to largely improve the treatment performance of a fleet of OSTs. We present a management strategy to reduce undetected failures drastically and thereby diminish negative impacts on environmental and human health.
\end{abstract}




\section{Introduction}

Water is one of the lifelines flowing through growing cities yet dwindling water resources require a substantial increase in water efficiency. One option is by developing modular systems of on-site wastewater treatment plants (OST), which can have a high local reuse potential. ${ }^{1-3}$ Such a paradigm shift from network based wastewater management systems towards more modular systems adapted to future challenges is urgently needed, as called for by Larsen et al. ${ }^{4}$ Decreasing the inflexibility of highly networked infrastructure and investment-intensive systems will increase planning and operating flexibility ${ }^{5}$ and is another reason to study OST systems. Considering the approximately 700 billion USD annual investment needs for water and wastewater infrastructure in OECD countries, ${ }^{6}$ there is abundant potential for rethinking the current paradigm. Eggimann et al. ${ }^{7}$ estimate the European market potential of OST solutions around $10 \%$ to $20 \%$ of the population, as at current cost level they are predominantly cost effective in less densely populated areas..$^{8-10}$ OSTs have a vast potential to complement centralised wastewater treatment plants (WWTP). However, they are still seen as a stopgap solution because of their unsatisfactory performance ${ }^{11}$ partly because they lack advanced performance monitoring, feedback control and performance quantification. ${ }^{12}$

To guarantee environmental and human health, a fleet of OSTs needs to be able to achieve a defined performance level in a verifiable manner from a systems perspective. Only few measurement campaigns assess the performance of OSTs by analysing grab samples, ${ }^{13-16}$ even less use online sensors for close to continuous measurements. ${ }^{17-19}$ Thus, information to characterise performance, OST reliability and failure rates remain sparse, despite calls for centralised remote monitoring and control of OSTs issued already in 1998.20 Therefore, a decisive factor for successful implementation of OSTs is an online monitoring concept. ${ }^{21-23}$

Olsson (2013) ${ }^{12}$ states that OSTs need less accurate sensors than centralized WWTP without quantifying these requirements. Hug and Maurer (2012) ${ }^{24}$ showed that a discrepancy between real and measured performance can be expected when monitoring OSTs. Schneider et al. ${ }^{25}$ suggested a soft sensor based on signals from unmaintained physical sensors, which solves the issue of labor-intensive maintenance on the costs of measurement accuracy. Soft-sensor accuracy (all true classifications divided by the total) has been identified to be $0.83-0.85$ based on four $\mathrm{pH}$ sensors, as well as 0.80 and 0.85 for two dissolved oxygen sensors. ${ }^{26}$ However, how the inaccuracy of this approach influences the performance of an entire fleet and its performance quantification is so far unknown.

In this article, we aim to fill this gap by investigating the impact of a monitoring concept based on the observation of such inaccurate soft sensors on the real treatment performance of a fleet of OSTs and how well this real performance can be quantified despite the inaccuracy of the sensors. Therefore, we developed a novel stochastic model to link monitoring, intervention intensity, and OST reliability and tested it with synthetic data. The following questions are addressed by making use of this model:

Q1: How do soft-sensor accuracy, reliability of OSTs, and alarm management influence treatment performance and number of required interventions?

Q2: How much can the performance of a system or fleet of OSTs be improved with online monitoring? 
Q3: How accurately can real system performance be quantified by observation with inaccurate soft sensors?

\section{Methods}

\subsection{Model concept}

The goal of the article is to estimate accuracy requirements on soft sensors to minimise effects on environmental and human health by unobserved malfunctions due to insufficient monitoring of OSTs. Therefore, we developed a model similar to a Petri net with aging tokens. ${ }^{27,28}$ The model quantitatively connects the different elements needed to operate and supervise a fleet of spatially distributed OST. The model output is an estimation of the effects of sensor inaccuracy on the treatment performance and quantification of this performance of a fleet of OSTs.

The model consists of five modules (see Figure 1): In the failure module (I), the state of the unit is simulated, e.g. failure occurrence or the effect of a unit repair (e.g. fully functioning immediately after maintenance or not). The performance module (II) quantifies the performance based on information about the state of the OST from the failure module. The monitoring module (III) represents all available observations (by soft sensors as well as by human intervention). This information is used to quantify observable system performance and serves as the basis for testing different types of alarm management in order to improve the systems treatment performance. The alarm management module (IV) defines which OSTs are flagged for intervention (maintenance, service). The intervention module (V) defines the frequency and types of intervention to be performed.

The presented model has two main applications. Firstly, to test monitoring strategies based on synthetic real performance, which provides support for system design decisions, such as balancing the costs of investment and maintenance for more accurate soft sensors against the costs of interventions caused by false alarms. Secondly, once sensors are installed in a system of OSTs, the presented model can help to estimate an observed performance that is as close to the real treatment performance as possible. The success of a strategy is measured by the relative improvement of the real performance, the number of interventions required (cost), and the difference between the observed and real performance. 


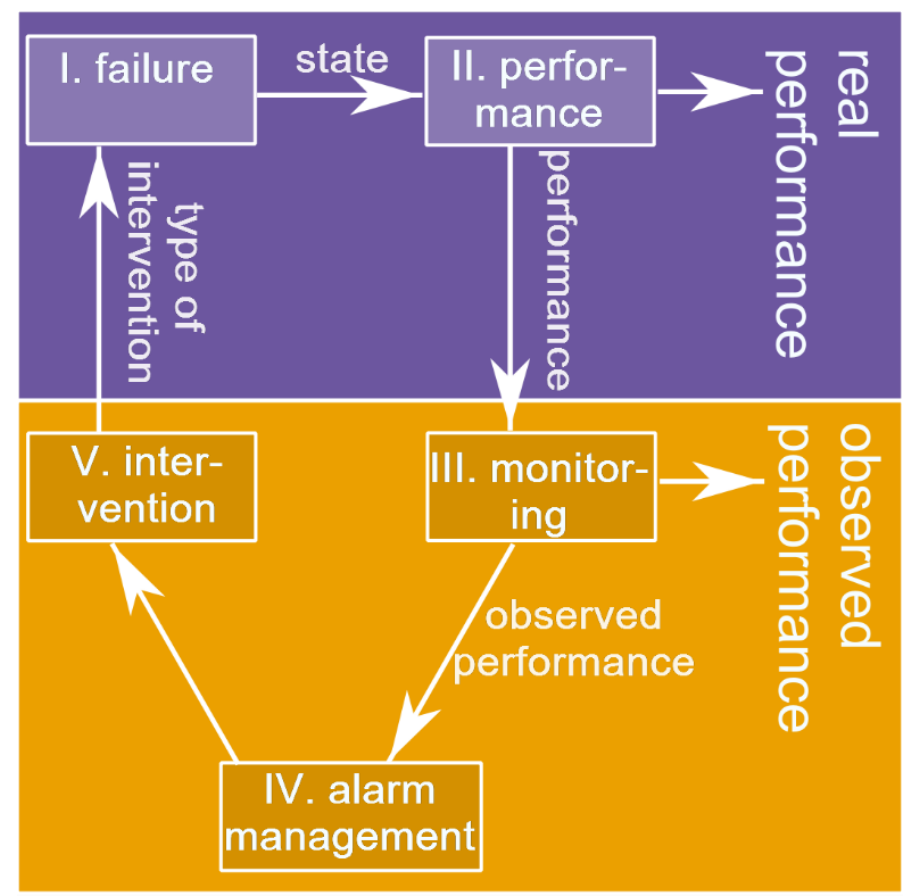

Figure 1: Systematic representation of the treatment performance model. I. and II. represent the only partially observable real treatment performance, III., IV., and V. the observable parts. The arrows indicate what information is transferred from one module to another.

\subsubsection{Real performance}

The real performance is described in two modules: the failure module (I) and the performance module (II). Together they mimic the underlying real performance of the individual OSTs, from which the real performance of the entire fleet of OSTs is quantified.

The failure module (I) provides the operational state of a unit. This can range from the normal operational state to technical malfunctions to breakdown of the biology due to a user error. We opted for a simplified binary representation: The state is labelled as 'up' for normal operation, otherwise 'down' for failures. A failure state is the most relevant and can be categorised, for example, as reversible or irreversible. Reversible failures are faults from which the OST recovers without intervention (e.g. a minor toxic shock), while irreversible failures can only be fixed through intervention (e.g. broken aeration equipment). The failure module is influenced by the intervention module and in turn influences the performance (see Figure 1).

Based on the OST state communicated from the failure module, the performance module (II) estimates the real performance of a specific unit in the fleet. Performance can be defined in different terms, but the real and the observed performance (derived from the monitoring module) should be compatible.

\subsubsection{Observed performance}

The observed performance characterises the operational part of an OST system and consists of three modules:

The monitoring module (III) represents the available information about the real treatment performance and includes data transmission. The observed information might stem from soft sensors but also human observation, e.g. by inspectors or local operators. The module output is the observed performance of 
the OST and builds the basis for alarm management and the observed system performance quantification.

The alarm management module (IV) aggregates and interprets information from the monitoring module on the level of individual OST's. The output of this module is a flag on units which need an intervention. ${ }^{29}$ The rules for flagging depend on the expected reliability of the observed performance and therefore on the accuracy of the soft sensor. If the soft sensor is inaccurate, for example, the observed state can be aggregated over time to provide enough certainty to trigger the threshold defined in this module (see Section 2.2 for the implementation). The alarms can be labelled, for example, with different flags for different levels of intervention urgency. Flags are used as a basis for the intervention module.

The intervention module $(\mathrm{V})$ defines the different types of interventions, the effort to carry out the intervention, and the effect the intervention has on the state of the unit defined in the failure module. Possible interventions are regular servicing (decreasing the failure probability) or maintenance called for by the alarm management module. In the simplest case, the intervention module just issues a maintenance intervention whenever an OST is flagged by the alarm module. Further examples of rules, which we did not implement - technicians are sent out depending on the state of the surrounding OSTs by a shortest path algorithm, or the observed systems performance could be used as a rule for the intervention module (if the system is in a critical state, interventions are carried out faster).

\subsection{Model implementation}

The model is implemented in the simplest way we could think of by still addressing the questions presented in the introduction. In every time step, the model passes through all modules in Figure 1. A time step is defined as one sequencing batch reactor (SBR) cycle that is assumed to last one day and is the frequency that new information becomes available from monitoring. The model is implemented using Python $3^{30}$ and is available under a GNU common license (Schneider ${ }^{31}$ on https://gitlab.com/Viraja/systemperformance/) and is based on Hug and Maurer (2012). ${ }^{24}$

Failure module (I): The failure module defines when the state of the OST changes. For this article, we have chosen a highly simplified implementation, where the OST state is characterised as a binary state. An 'up' state means that the unit is fully functioning and a 'down' state indicates that the unit is in a failure situation. 'Fully functioning' assumes that the unit functions per design criteria. We implemented a failure situation as an irreversible event having a high probability for insufficient performance and for resolution occurring only with an intervention (maintenance). This means that an 'up' state can change to a 'down' state, but not vice versa without an intervention executed by the intervention module.

For our implementation, we used reliability theory, assuming that the failures are stochastic ${ }^{32}$ and chose a Weibull distributed failure rate. ${ }^{33}$ The Weibull distribution if often used in infrastructure management; however, other distributions could be used as well. To check if an OST which is 'up' fails in the current time step, a random number between 0 and 1 is drawn from a uniform distribution. This number is compared with the Weibull distributed hazard rate in eq 1 . If the random number is smaller than the hazard rate, the OST state is changed to 'down'. If the random number is greater than or equal to the hazard rate, the state stays unchanged. 
$\operatorname{hazard} \operatorname{rate}(t)=\frac{f(t)}{1-\int_{0}^{t} f(t) d t}$ or hazard rate $(t, k, \lambda)=\frac{k}{\lambda}\left(\frac{t}{\lambda}\right)^{k-1}$

Unit age is represented with $t, \lambda$ is the scale factor, $k$ the shape parameter, and $f(t)$ is the Weibull probability density function. For our screening, we kept the shape parameter $k$ constant at 2 (as in a previous study ${ }^{24}$ ) and varied the scale factor $\lambda$ in a grid search to cover a wide range of mean unit survival times, as this is a parameter to which the performance is sensitive. For other failure modes - e.g. periods without inflow, installation or user errors - probably follow a different distribution and would need the implementation of additional failure probability functions.

Performance module (II): In the current implementation an 'up' state of the failure module translates into an 'up' state of the performance model. Such an 'up' state would for example mean a complete nitrification. Likewise, a 'down' state of the failure module translates into a 'down' performance.

Monitoring module (III): Analog to the failure and performance module, the monitoring module also records a binary state ('up' and 'down') based on the information from the performance module with a defined accuracy between 0.5 and 1.0. A soft sensor with an accuracy of 0.5 is a random soft sensor; one with an accuracy of 1.0 represents a perfect soft sensor. The soft-sensor accuracy might be defined differently for detecting an 'up' or 'down' performance. Consequently, the following sensing outcomes are possible:

- True positive (TP): The soft sensor indicates 'up' and the real performance is 'up'.

- False negative (FN): The soft sensor indicates 'down' and the real performance is 'up'. The probability for FN outcome is 1 minus the TP. The FN potentially causes false alarms.

- True negative (TN): The soft sensor indicates 'down' and the real performance is 'down'.

- False positive (FP): The soft sensor indicates 'up' and the real performance is 'down'. The probability for this outcome is 1 minus the TN. The FP represents undetected failures.

The soft-sensor measurement is determined stochastically, i.e., for every time step, a random number between 0 and 1 is drawn from a uniform distribution. This random number is then compared with the sensitivity (eq 2), if the performance is 'up', and with the specificity (eq 3), if the performance is 'down'. If the random number is smaller than the sensitivity, respectively the specificity, the prediction is true, otherwise false.

$$
\begin{aligned}
& \text { sensitivity }=\frac{T P}{F N+T P} \\
& \text { specificity }=\frac{T N}{T N+F P}
\end{aligned}
$$

This implementation mimics the soft sensors developed by Schneider et al. ${ }^{26}$ These soft sensors predict the completeness of ammonium oxidation of a biological process in an SBR based on signals of unmaintained $\mathrm{pH}$ and dissolved oxygen sensors (with 'up' and 'down' defined as an effluent ammonium effluent concentration below and above $1 \mathrm{gNm}^{-3}$ ). For the $\mathrm{pH}$-based soft sensor, a specificity of 0.98 and a sensitivity of 0.71 were identified through experimental validation under realistic conditions. Furthermore, we assumed for this model implementation that a complete soft-sensor failure either is noticed immediately or doesn't occur, based on our experience ${ }^{26,34}$ where more than ten commercial $\mathrm{pH}$ sensors were tested. 
The alarm management module (IV) converts the information from the monitoring module into a label communicating an action to be taken. The simplest approach is to flag a unit as needing an intervention as soon as a soft sensor identifies the performance as negative. Depending on the soft-sensor accuracy, this might result in many false alarms. An approach to improve confidence is to wait for $n$ consecutive cycles labelled negative before flagging an OST for an intervention. A more elaborate method uses previously identified soft-sensor accuracy to calculate the likelihood of the unit being 'down' based on the given signal. As soon as this likelihood has surpassed a threshold (we used $98 \%$ as a boundary close to, but lower than $100 \%$ ), an intervention is periodic for the corresponding unit. To estimate the likelihood of the unit being 'down', we calculate the conditional probability based on prior information with a sequential probability ratio test: ${ }^{35}$

$$
P_{t}(\text { perf }=\text { down } \mid \text { signal }=\text { negative })=\frac{P_{t-1}(\text { perf }=\text { down }) \cdot T N}{P_{t-1}(\text { perf } f=\text { down }) \cdot T N+\left(1-P_{t-1}(\text { perf } f=\text { down })\right) \cdot(1-T P)}
$$

And correspondingly:

$P_{t}($ perf $=$ down $\mid$ signal $=$ positive $)=\frac{P_{t-1}(\text { perf }=\text { down }) \cdot(1-T N)}{P_{t-1}(\text { perf }=\text { down }) \cdot(1-T N)+\left(1-P_{t-1}(\text { perf } f=\text { down })\right) \cdot T P}$

$P\left(\right.$ perf $_{\text {time }}=$ down $\mid$ signal time $_{\text {ime }}$ negative $)$ reads the following way: The probability that the real performance of an OST is 'down' given that the soft sensor shows a negative detection at time t based on the likelihood from the last time step ( $\mathrm{t}-1)$, which is the prior. The likelihood that an OST is 'down', is updated for every time step using eq 4 and 5 and the likelihood from the previous time step. To avoid numerical issues and a memory effect, we limit the likelihood interval to $[0.001,0.999]$ with a simple stopping rule. Intervention module $(V)$ : The intervention is implemented in the way that every time a flag is raised in the alarm module an intervention is triggered. To minimise model complexity, we do not distinguish between different types of interventions nor consider spatial efficiencies. The assumption is that the intervention always repairs the OST. This means that if the unit state is 'down' it will be set back to 'up' (functioning) after an intervention. Via the failure module, the real performance is also set to 'up'. Furthermore, we did not implement an impact of an unnecessary (if the state was 'up' before the intervention) intervention on the failure probability. In addition to demand-driven interventions, a periodic (timedriven) inspection can be requested. Regular inspections act as a comparison of the real performance between a periodic and a demand-driven inspection and repairs a unit with 'down' status in the same manner as a demand-driven intervention.

\subsubsection{System performance}

The real system performance is calculated based on information from the performance module, and correspondingly, the observed system performance is based on the information from the monitoring module. The average real performance of the system is simply the mean of the performance of all individual OSTs in the system. The average observed performance is estimated, by calculating the mean of all soft-sensor measurements in the monitoring module (III).

\subsubsection{Operational costs}

Differences in operational costs are simply represented as number of interventions. 


\section{Results and Discussion}

OSTs have a large potential to change wastewater from "waste" into a resource and enable locally appropriate recovery solutions. ${ }^{36}$ However, without appropriate monitoring an inferior performance in comparison to centralised approaches are to be expected. The results in this article obtained by stochastic modelling shows that monitoring even with inaccurate sensors improves the treatment performance significantly, but also highlights that the quantification of this performance requires accurate sensors.

\subsection{Q1: How do soft-sensor accuracy, reliability of OSTs, and alarm management influence treatment performance and number of required interventions?}

Figure 2 shows the average fraction of time that a single OST's performance is 'up' (functioning) over a ten-year time frame. Every pixel represents an OST modelled with reliability (the mean survival time) as indicated on the $\mathrm{x}$-axis and the soft-sensor accuracy (sensitivity and specificity are identical) as indicated on the y-axis. As the target is to have both high performance (Figure 2) and a low number of interventions (Figure 3) these two figures need to be considered jointly. To better highlight the results, we highlight two meaningful regions in the subplots of Figure 2: $Y$ high performance and $X$ low performance. Similarly, Figure 3 is marked by two regions: A low number of interventions (feasible) and B high number of interventions (infeasible). These areas are used purely to guide the reader through the results and discussion.

In Figure 2a, an intervention is issued as soon as the soft sensor shows a negative signal. Not surprisingly, this leads to a very high number of units in the 'up' state (showing the performance per design specification). Interestingly, even under these very intervention-intensive circumstances, the performance is not at $100 \%$ for very low mean survival time and low sensor accuracy. Figure 3 a shows the high number of interventions - and therefore costs - this alarm management causes. Only with very accurate soft sensors (>98\%) and elevated OST reliability does the intervention demand decrease to a reasonably low level. 

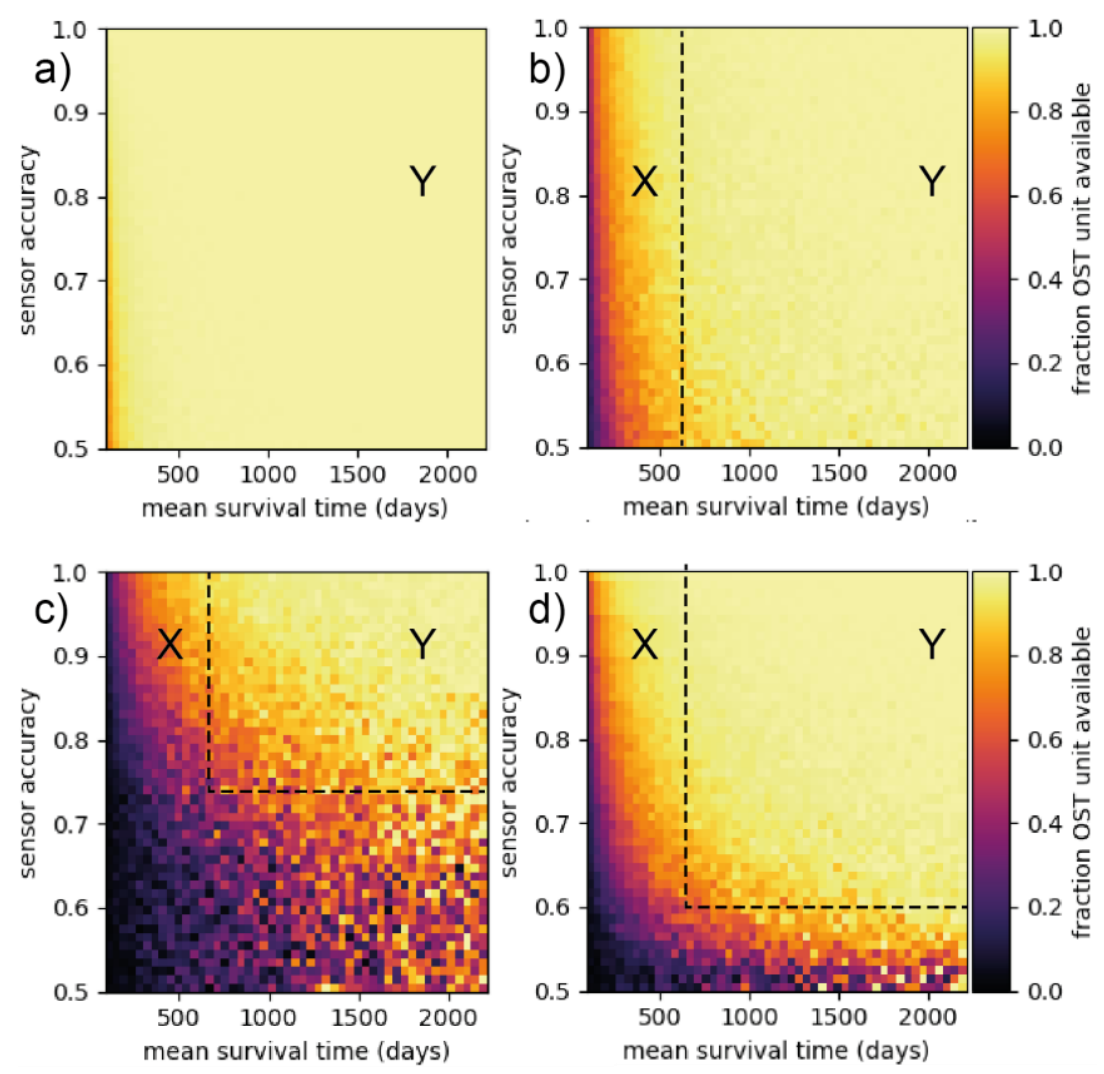

Figure 2: Fraction of time the real performance of an OST is 'up'. Every pixel represents an OST with a specific sensor accuracy and a failure rate averaged over 10 years. The difference between the four subplots is the alarm management; an intervention is issued after the soft sensor shows negative for a) one day, b) four consecutive days, c) 14 consecutive days, d) conditional probability of a failure $>98 \%$. The axis is from 88 to 2216 days. The subplots are schematically divided into two sections: ( $Y$ ) high performance, $(X)$ low performance. 

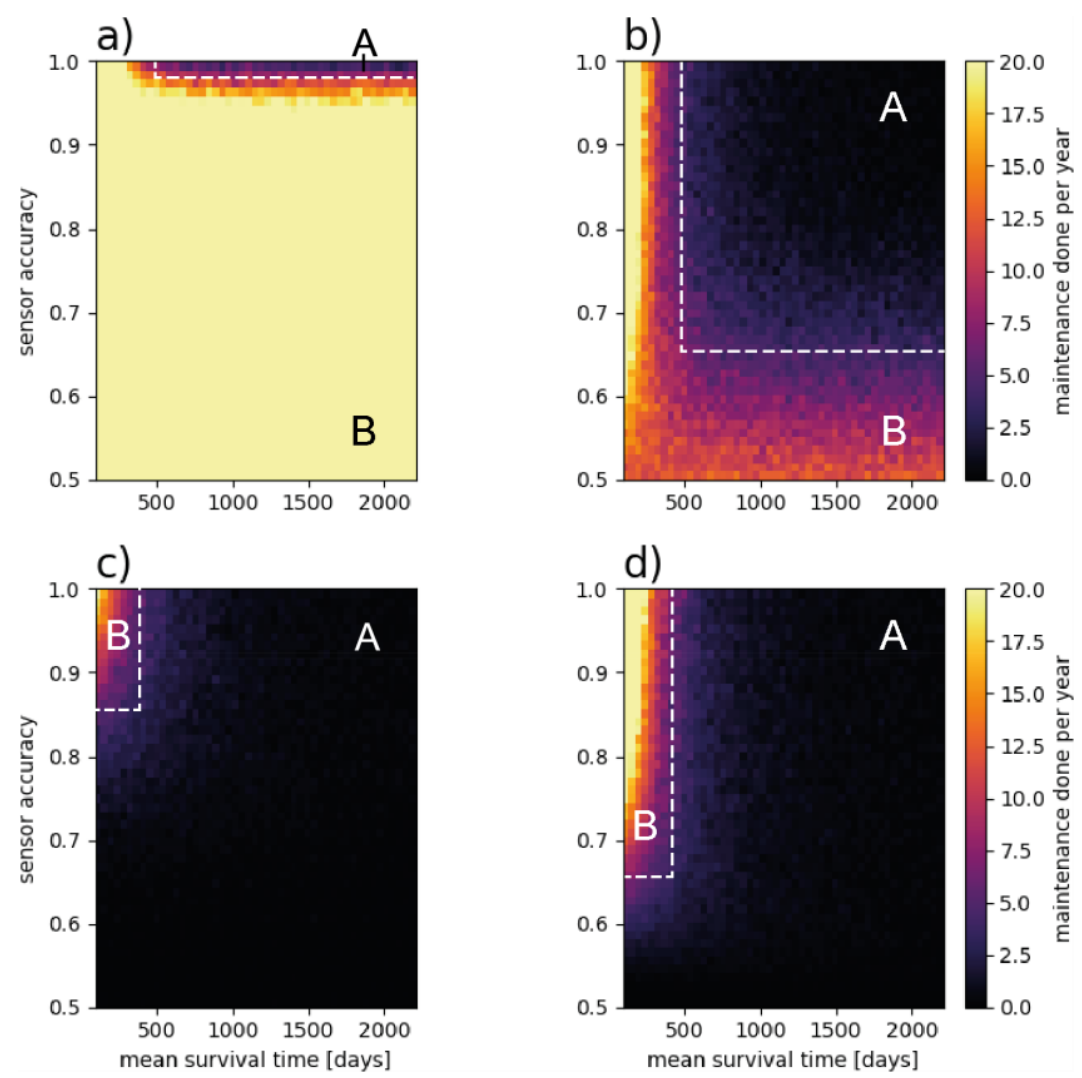

Figure 3: The interventions needed per year for the performance shown in Figure 2. Every pixel is the mean number of interventions over 10 year for one individual OST. The difference between the four subplots is the alarm management; an intervention is issued after the soft sensor is showing negative for a) one cycle, b) four consecutive cycles, c) 14 consecutive cycles, d) conditional probability of a failure $>98 \%$. The subplots are schematically divided by the number of interventions per year $(A)$ feasible and $(B)$ unfeasible.

Figure 2 show the impact of alarm management on the 'up' time of the unit, by simply increasing the number of consecutive negative signals an intervention needs in order to be triggered: a) one b) four, and c) 14 consecutive time steps. A small delay (four time steps) of the intervention, as in Figure $2 \mathrm{~b}$, leads to a substantial decrease of 'up' time for units with low reliability. But from Figure 3b, a substantial decrease in the number of interventions can be seen for much of the parameter domain. This trend continues in Figure $3 \mathrm{c}$ with the difference that the effect of low accuracy sensors can be clearly seen in Figure 2c. Soft sensors with low or intermediate accuracy have a low probability of showing a series of 14 consecutive negative signals and therefore are prone to large intervention delays - even when the 'down' performance is very clear, like in our example. The critical value for consecutive negative signals therefore lies somewhere between 4 and 14 days. Figure $2 d$ shows the impact of more sophisticated alarm management based on the confidence in the monitoring signal. Interventions are triggered if the estimated likelihood (eq 4 and 5) of 'down' state of a unit exceeds 0.98 . This approach assumes that the soft-sensor accuracy is known and links it with the alarm management. High 'up' time for units is achieved for all but the least reliable units and with medium or higher accuracy soft sensors.

OSTs optimally are operated somewhere in the intersection of areas $\mathrm{Y}$ (Figure 2) and A (Figure 3) ( $\mathrm{Y} \cap$ A), where the treatment performance is high while the number of interventions remains low. In the following we refer to a), b), c), and d) and mean the management strategy behind the related subplot in Figure 2 and Figure 3 and discuss which factors restrict $Y \cap A$. 
$\mathrm{Y} \cap \mathrm{A}$ of $\mathrm{a}$ ) is restricted by $\mathrm{A}$. An alarm management that triggers an intervention as soon as the sensor indicates a negative signal will lead to many $\mathrm{FN}$ and therefore high costs, which a large amount of interventions would cause. The exception are nearly perfect sensors with more than $98 \%$ accuracy. Conversely, $\mathrm{Y} \cap \mathrm{A}$ for management strategy $\mathrm{c}$ ) is only curtailed by $\mathrm{Y}$, the treatment performance displayed in Figure 2c, suggesting that not enough interventions are triggered with this alarm management. In this case, the threshold used to trigger an alarm is set too high, leading to delayed inspections and reduced performance.

The most successful alarm management strategies are b) and d). Interestingly, both strategies produce a very similar resulting $Y \cap A$ area, although they have different underlying causes. For $b$ ) high number of interventions are needed (Figure 3, area A), while the performance is high for any sensor accuracy between 0.5 and 1.0 (Figure 2, area Y). For d) the opposite can be observed: the number of interventions is low for all sensor accuracies, but for sensor accuracies below 0.6 the treatment performance drops.

Therefore, for an individual OST, if the sensitivity and specificity are identical, a sensor accuracy of at least 0.7 , in combination with appropriate alarm management, is required to be able to achieve treatment performances above $90 \%$.

Assuming that both $b$ ) and d) are preferable alarm strategies, we can learn that a minimal OST reliability is required or a replacement strategy ${ }^{37}$ needs to be set in place to avoid the high number of failures at the end-of-life of the unit. In b) and d), this threshold is very roughly around a one year mean survival time if the failure follows a Weibull distribution (eq 5). Soft sensors have no such clear threshold because we assume that they are replaced frequently enough to not reach the end-of-life phase.

\subsection{Q2: How much can the performance of a system or fleet of OSTs be improved with online monitoring?}

The goal is to quantify how much the real system performance improves due to demand-driven interventions based on online monitoring when compared to the same amount of periodic time-driven maintenance interventions. This periodic maintenance schemes without online monitoring is currently the standard management strategy for OSTs in the real world practice. ${ }^{38-41}$ Typical intervals are in Australia (4 times per year ${ }^{39,42}$ ), Germany (2-3 times per year $\left.{ }^{43-45}\right)$, Japan ( $\geq 1$ time per year $\left.{ }^{40}\right)$, and Switzerland $\left(\geq 1\right.$ time per year $\left.{ }^{38}\right)$, therefore 1-4 interventions per year currently seem a feasible range of interventions for OSTs. To answer the question (Q2) we modelled a system with 10000 OSTs for a period of ten years with reliabilities (random uniform distribution of $\lambda$ between 400 and 2200). Three base scenarios were modelled for $\mathrm{N}$ numbers of interventions per year i) $\mathrm{N}=0$ (without any intervention), ii) $\mathrm{N}=1$, and iii) $\mathrm{N}=3$. The output of the modelling is the mean availability (up label) of the 10000 OSTs over a period of 10 years. The results as mean availability are i) 0.31 of the time (31\% 'up' time of the 10000 OSTs out of $10^{*} 365$ days) for $\mathrm{N}=0$, ii) 0.66 for $\mathrm{N}=1$, and iii) 0.83 for $\mathrm{N}=3$. These results are indicated in Figure 4 as horizontal, dashed lines.

Figure 4 further shows the influence of different soft-sensor accuracies on the real treatment performance as well as how accurate the real performance is observed. The modelled observed performance improves nearly linearly with the soft-sensor accuracy. This is the behaviour which we would expect in a system with a close to $100 \%$ real treatment performance, the observed performance in this special 
case directly reflects the soft-sensor accuracy. The real performance is always above $90 \%$ even with inaccurate ( $50 \%$ as a random sensor) soft sensors. However, the high performance despite low sensor accuracy comes at a cost of maintenance frequency. 12 interventions cause three times as much costs as the maximum of 4 interventions found in the real-world. It can be read from Figure 4 that the minimal acceptable soft-sensor accuracy is approximately 0.7 with 3 interventions. The modelled treatment performance for a 0.7 soft-sensor accuracy is $97 \%$ (with $0.12 \%$ variance, see supporting information). The previously developed $\mathrm{pH}$-based soft sensors have an overall accuracy of $0.80-0.85,{ }^{26}$ well above this minimum of 0.7 , which is required to improve the treatment performance of a system of OSTs. This suggests that a wide implementation of these real-world sensors would significantly improve the treatment performance. Figure 4 suggests an improved treatment performance from $66 \%$ (1 periodic intervention) to $98 \%$ (with 1-2 demand-driven interventions with 0.8 soft-sensor accuracy). Therefore, when changing from the status quo (regular, periodic interventions), towards measurement-based, demanddriven interventions even with inaccurate soft sensors, the system potentially shifts from a mediocre to a decent, real performance.

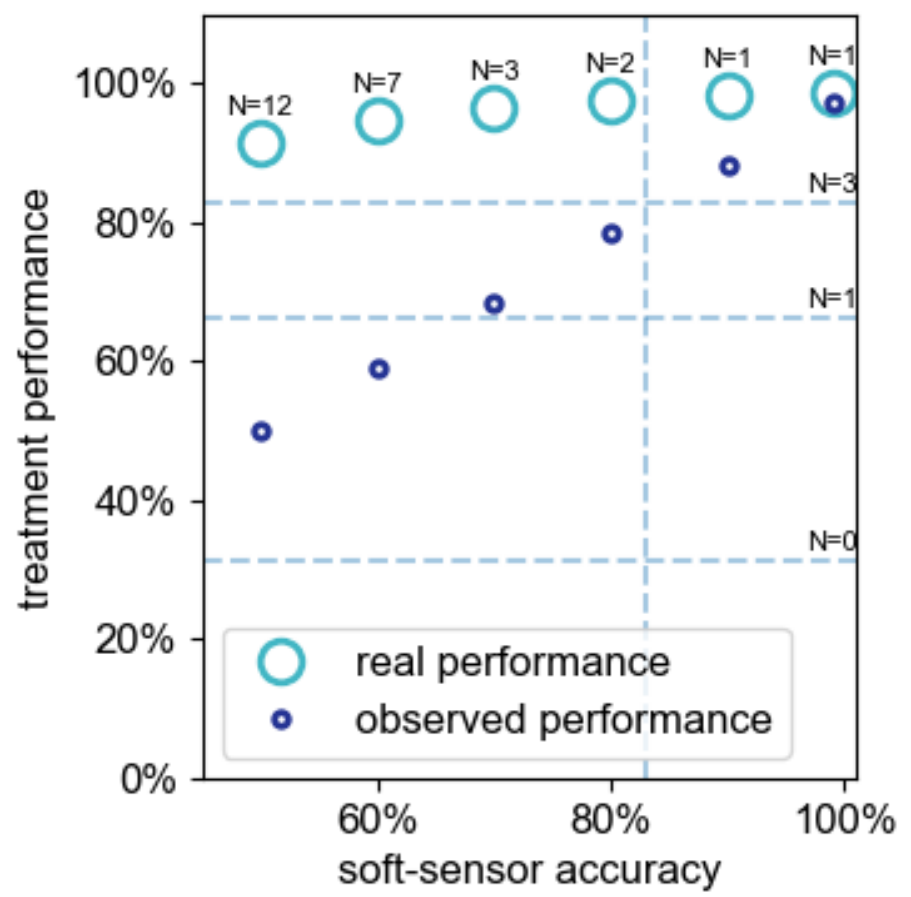

Figure 4: Comparison of real and observed performance for different soft-sensor accuracies. The dashed lines indicate the treatment performance for one yearly periodic intervention (horizontal) and the accuracy of the existing real-world soft sensor (vertical). $N$ is the number of interventions to reach the indicated performance. The reliability $\lambda$ is between 400 and 2200.

\subsection{Q3: How accurately can real system performance be quantified by observation with inaccurate soft sensors?}

Figure 4 shows the average real performance for a stochastically modelled system of 10000 OSTs, as well as the observed system performance derived from monitoring with inaccurate sensors, and the average number of interventions per unit per year. The observed performance deviates from the real performance depending on the sensor accuracy and is - except in the extreme case of a nearly perfect sensor - substantially lower than the real performance. Table 1 shows two essential but diverging aspects of sensor accuracy. The modelling results imply, that an optimisation for specificity improves 
the treatment performance of an OST, but reduces the information content on the performance. Monitoring with high specificity is especially relevant for the high variability such small OSTs generally encounter. ${ }^{13}$ Therefore, the strong deviation between real and observed performance in Figure 4 is increased by the parametrisation of the soft sensor; The existing soft sensors are optimised for specificity ${ }^{26}$. The benefit of this conservative approach is that it results in more reliable information for alarm management, which in turn improves the real treatment performance. However, this specificity-optimised soft sensor contributes less information for the observed performance.

Table 1: Influence of soft-sensor specificity and sensitivity and reliability on mean real performance, the mean observed performance, and mean number of interventions per unit per year for a system of 10000 OSTs. "as Figure 4" uses the same random uniformly distributed $\lambda$ as Figure 4 (400 and 2200), 'low' (100 to 400), 'high' (800 to 2200).

\begin{tabular}{|l|l|c|c|c|}
\hline \multirow{2}{*}{$\begin{array}{l}\text { sensor } \\
\text { accuracy }\end{array}$} & OST reliability & $\begin{array}{c}\text { mean real perfor- } \\
\text { mance }\end{array}$ & $\begin{array}{c}\text { mean observed per- } \\
\text { formance }\end{array}$ & $\begin{array}{c}\text { mean number of in- } \\
\text { terventions }\end{array}$ \\
\hline \multirow{2}{*}{$\begin{array}{l}\text { specificity 0.98, } \\
\text { sensitivity 0.7 }\end{array}$} & as Figure 4 & 0.99 & 0.69 & 3.5 \\
\cline { 2 - 5 } & low & 0.82 & 0.54 & 23 \\
\hline \multirow{2}{*}{$\begin{array}{l}\text { specificity 0.7, } \\
\text { sensitivity 0.98 }\end{array}$} & As Figure 4 & 0.99 & 0.71 & 2.5 \\
\cline { 2 - 5 } & low & 0.96 & 0.95 & 1.4 \\
\cline { 2 - 5 } & high & 0.64 & 0.71 & 0.80 \\
\hline
\end{tabular}

The results of the modelling clearly suggest that the soft sensor should be used with two different sets of optimised parameters; one geared toward high specificity for the alarm management and one toward high sensitivity to quantify the system performance. Both can be done with the same calibration data as an input. Realising this possible double use of data to obtain different information, is an important change in mind-set and a strength of the soft-sensor approach.

\subsection{Alarm management}

Two alarm strategies have been identified as preferable to the other two investigated strategies. One is very simple, by waiting for four consecutive negative cycles; the other uses a conditional probability that depends on sensor accuracy. The latter is the more elaborate method. However, it has two disadvantages: Firstly, an error in the estimated sensor accuracy could lead to an inadequate system performance quantification. Secondly, if the accuracy is lower than estimated, the performance is lower, but the number of interventions would not raise as required because of the overestimated accuracy (Figure $2 \mathrm{~d}$ and Figure $3 \mathrm{~d}$ ). In reality, if a low performance of an individual OST occurs due to a wrong estimation of sensor accuracy, the low performance would most probably not be discovered because performance is estimated based on this same inaccurate sensor. In contrast, for the simple approach a sensor with a lower accuracy would simply cause more interventions (Figure $2 b$ and Figure $3 b$ ). A rising number of interventions is likely to be spotted by an operator. This shows that when choosing a preferred alarm management, ease of identification of an unfavourable state of the system should also be considered.

\subsection{Simplifications and assumptions}

The presented model can be extended to quantify OST systems more realistically. For further studies, improving the binary performance model with more differentiated failure modes (e.g. biology failure due to prolonged periods without influent) and effective performance distributions would give a more detailed quantitative estimation about the achievable performance of real OST systems. 
Similarly, the presented alarm management implementation is highly simplified and ignores much potential to analyse the monitoring data. E.g. currently, the signals from the sensors are treated as statistically independent. Possible improvements include considering machine learning algorithms for anomaly detection in the online time series - or spatially optimising an intervention strategy that also considers OST locations. However, the current version of the model provides a result that indicates the minimal requirements that we expect for such a monitoring concept to work.

\subsection{Comparison of the system performance between centralised WWTP and OSTs}

When comparing OST and centralised systems, it is remarkable that even for centralised systems many unknowns about the real treatment performance remain; Firstly, losses via combined sewer overflows or faulty connections are only partly monitored, ${ }^{46}$ e.g. in Switzerland $2.7 \%$ of the yearly dry-weather flow is estimated to be discharged untreated during rain events via combined sewer overflows. ${ }^{46}$ Secondly, losses in sewers due to suboptimal infrastructure management are even less known. In Europe, sewer losses are estimated to be from 5-20 \%, ${ }^{47}$ which is low compared to the large amount of $77 \%$, for example, estimated for Vietnam. ${ }^{48}$ Thirdly, the inflow concentrations of ammonium to OSTs can be very high. ${ }^{14,25}$

We therefore argue that the focus on high treatment performance of one unit (instead of system performance) underestimates the comparative advantage that distributed systems can have over centralised systems. These untreated losses indicate that centralised systems only exceed $90 \%$ elimination performance in exceptional situations. This article shows that OST systems with adequate monitoring have the potential to achieve these performance levels as well. We believe that this justifies the need for more research into remote operation and monitoring of decentralized, modular, and unstaffed wastewater treatment systems.

Lastly, we want to emphasise the importance of evaluating the environmental impact at the system perspective. Without professional monitoring, maintenance and repair, no technical system works properly. ${ }^{49}$ This applies to OST and centralised systems. Therefore, for further scale-up of OST systems, centralised management ${ }^{50}$ and appropriate monitoring strategies are essential.

\section{Abbreviations}

OST on-site wastewater treatment plant

SBR sequencing batch reactor

WWTP wastewater treatment plant

\section{Acknowledgements}

We would like to thank Peter Vanrolleghem his detailed feedback; Andreas Scheidegger, Philipp Beutler, Rachel Barrett, Omar Wani, Liliane Manny, Lena Mutzner, and Matthew Moy de Vitry for input, and Joan de Vitry-Moy for language advice. MYS acknowledges support from the JSPS (Grant number GR19105 and P20763). 


\section{Author contributions}

MYS did the modelling and led the project and the writing of the article. MM and KV provided ideas and expertise. HH provided experience on OSTs in Japan. MM and KV supervised the work. All authors contributed to the writing of this document.

\section{Funding Sources}

MYS received funding from the Japanese Society for Promotion of Science (JSPS) Grant GR19105 and P20763.

\section{References}

(1) Gikas, P.; Tchobanoglous, G. The Role of Satellite and Decentralized Strategies in Water Resources Management. J. Environ. Manage. 2009, 90 (1), 144-152. https://doi.org/10.1016/j.jenvman.2007.08.016.

(2) Hoffmann, S.; Feldmann, U.; Bach, P. M.; Binz, C.; Farrelly, M.; Frantzeskaki, N.; Hiessl, H.; Inauen, J.; Larsen, T. A.; Lienert, J.; Londong, J.; Lüthi, C.; Maurer, M.; Mitchell, C.; Morgenroth, E.; Nelson, K. L.; Scholten, L.; Truffer, B.; Udert, K. M. A Research Agenda for the Future of Urban Water Management: Exploring the Potential of Non-Grid, Small-Grid, and Hybrid Solutions. Environ. Sci. Technol. 2020, acs.est.9b05222. https://doi.org/10.1021/acs.est.9b05222.

(3) Langergraber, G.; Muellegger, E. Ecological Sanitation-a Way to Solve Global Sanitation Problems? Environ. Int. 2005, 31 (3), 433-444. https://doi.org/10.1016/j.envint.2004.08.006.

(4) Larsen, T. A.; Hoffmann, S.; Lüthi, C.; Truffer, B.; Maurer, M. Emerging Solutions to the Water Challenges of an Urbanizing World. Science 2016, 352 (6288), 928-933. https://doi.org/10.1126/science.aad8641.

(5) Neumann, M. B.; Rieckermann, J.; Hug, T.; Gujer, W. Adaptation in Hindsight: Dynamics and Drivers Shaping Urban Wastewater Systems. J. Environ. Manage. 2015, 151, 404-415. https://doi.org/10.1016/j.jenvman.2014.12.047.

(6) Ashley, R.; Cashman, A. Infrastructure to 2030: Telecom, Land Transport, Water and Electricity; OECD; OECD Publishing: Paris, 2006.

(7) Eggimann, S.; Truffer, B.; Feldmann, U.; Maurer, M. Screening European Market Potentials for Small Modular Wastewater Treatment Systems - an Inroad to Sustainability Transitions in Urban Water Management? Land Use Policy 2018, 78, 711-725. https://doi.org/10.1016/j.landusepol.2018.07.031.

(8) Dahlgren, E.; Göçmen, C.; Lackner, K.; van Ryzin, G. Small Modular Infrastructure. Eng. Econ. 2013, 58 (4), 231-264. https://doi.org/10.1080/0013791X.2013.825038.

(9) Libralato, G.; Volpi Ghirardini, A.; Avezzù, F. To Centralise or to Decentralise: An Overview of the Most Recent Trends in Wastewater Treatment Management. J. Environ. Manage. 2012, 94 (1), 61-68. https://doi.org/10.1016/j.jenvman.2011.07.010.

(10) Massoud, M. A.; Tarhini, A.; Nasr, J. A. Decentralized Approaches to Wastewater Treatment and Management: Applicability in Developing Countries. J. Environ. Manage. 2009, 90 (1), 652-659. https://doi.org/10.1016/j.jenvman.2008.07.001.

(11) Moelants, N.; Janssen, G.; Smets, I.; Van Impe, J. Field Performance Assessment of Onsite Individual Wastewater Treatment Systems. Water Sci. Technol. 2008, 58 (1), 1-6. https://doi.org/10.2166/wst.2008.325.

(12) Olsson, G. Chapter 13: The Potential of Control and Monitoring. In Source Separiation and Decentralization for Wastewater Management edited by T. A. Larsen, K. M. Udert, J. Lienert; IWA Publishing: Londen, UK, 2013; pp 179-192.

(13) Lowe, K. S.; Tucholke, M. B.; Tomaras, J. M. B.; Conn, K.; Hoppe, C.; Drewes, J. E.; McCray, J. E.; Munakata-Marr, J. Influent Constituent Characteristics of the Modern Waste Stream from Single Sources; IWA Publishing, 2010. https://doi.org/10.2166/9781780403519.

(14) Lowe, K. S.; Tucholke, M. B.; Tomaras, J. M. B.; Conn, K.; Hoppe, C.; Drewes, J. E.; McCray, J. E.; Munakata-Marr, J. Influent Constituent Characteristics of the Modern Waste Stream from Single Sources: Final Report http://web.archive.org/web/20190216024758/http://www.decentralizedwater.org/research_project_04-DEC-1.asp (accessed 2020 -04 -28).

(15) Schranner, T. Kleinkläranlagen Bewähren Sich in Der Praxis (in German). Korresp. Abwasser Abfall 2014, 61 (8), 695-700. https://doi.org/10.3242/kae2014.08.003. 
(16) Klinger, M.; Ulrich, L.; Ramprasad, C.; Wolf, A. T.; Reynaud, N.; Narayan, A. S.; Siemsen, P.; Lüthi, C.; Philip, L. Technology, Implementation and Operation of Small-Scale Sanitation in India-PerformanceAnalysis and Policy Recommendations; 4S Project Report Vol. I; 2020.

(17) Straub, A. Einfache Messmethoden zur Charakterisierung sowie Maßnahmen zur Erhöhung der Zuverlässigkeit und Leistungsfähigkeit biologischer Kleinkläranlagen (in German). Schriftenreihe Siedlungswasserwirtschaft und Umwelt: Heft 17, Cottbus, 2008.

(18) Abegglen, C.; Joss, A.; McArdell, C. S.; Fink, G.; Schlüsener, M. P.; Ternes, T. A.; Siegrist, H. The Fate of Selected Micropollutants in a Single-House MBR. Water Res. 2009, 43 (7), 20362046. https://doi.org/10.1016/j.watres.2009.02.005.

(19) Schneider, M. Y.; Furrer, V.; Sprenger, E.; Sterkele, B.; Richter, S.; Rottermann, K. Data for: Benchmarking Soft-Sensors for Remote Monitoring of on-Site Wastewater Treatment Plants. 2020. https://doi.org/10.25678/000194.

(20) Crites, R.; Tchobanoglous, G. Small and Decentralized Wastewater Management Systems; McGraw-Hill Ser. in Water Resources and Environmental Engineering; McGraw-Hill: Boston, 1998.

(21) Coleman, J. J.; Abrams, I. J. Mathematical Model for Operational Readiness. Oper. Res. 1962, 10 (1), 126-138. https://doi.org/10.1287/opre.10.1.126.

(22) Rieger, L.; Vanrolleghem, P. A. MonEAU: A Platform for Water Quality Monitoring Networks. Water Sci. Technol. 2008, 57 (7), 1079-1086. https://doi.org/10.2166/wst.2008.135.

(23) Thomann, M.; Rieger, L.; Frommhold, S.; Siegrist, H.; Gujer, W. An Efficient Monitoring Concept with Control Charts for On-Line Sensors. In Water Science and Technology; 2002; Vol. 46, pp 107-116.

(24) Hug, T.; Maurer, M. Stochastic Modeling to Identify Requirements for Centralized Monitoring of Distributed Wastewater Treatment. Water Sci. Technol. 2012, 65 (6), 1067-1075. https://doi.org/10.2166/wst.2012.945.

(25) Schneider, M. Y.; Furrer, V.; Sprenger, E.; Carbajal, J. P.; Villez, K.; Maurer, M. Benchmarking Soft Sensors for Remote Monitoring of On-Site Wastewater Treatment Plants. Environ. Sci. Technol. 2020, 54 (17), 10840-10849. https://doi.org/10.1021/acs.est.9b07760.

(26) Schneider, M. Y.; Carbajal, J. P.; Furrer, V.; Sterkele, B.; Maurer, M.; Villez, K. Beyond Signal Quality: The Value of Unmaintained PH, Dissolved Oxygen, and Oxidation-Reduction Potential Sensors for Remote Performance Monitoring of on-Site Sequencing Batch Reactors. Water Res. 2019, 161, 639-651. https://doi.org/10.1016/j.watres.2019.06.007.

(27) Volovoi, V. System-Level Maintenance Policies via Stochastic Petri Nets with Aging Tokens. In 2007 Annual Reliability and Maintainability Symposium; 2007; pp 89-94. https://doi.org/10.1109/RAMS.2007.328049.

(28) Volovoi, V. Modeling of System Reliability Petri Nets with Aging Tokens. Reliab. Eng. Syst. Saf. 2004, 84 (2), 149-161. https://doi.org/10.1016/j.ress.2003.10.013.

(29) Arnold, M. W.; Darius, I. H. Alarm Management in Batch Process Control. ISA Trans. 1989, 28 (3), 33-40. https://doi.org/10.1016/0019-0578(89)90025-6.

(30) Van Rossum, G.; Drake, F. L. Python 3 Reference Manual; CreateSpace: Scotts Valley, CA, 2009.

(31) Schneider, M. Y. Systemperformance Module Python 3; https://gitlab.com/Viraja/systemperformance/; 2020.

(32) Barlow, R. E.; Proschan, F. Mathematical Theory of Reliability; Society for Industrial and Applied Mathematics, 1996. https://doi.org/10.1137/1.9781611971194.

(33) Weibull, W. A Statistical Distribution Function of Wide Application. ASME J. Appl. Mech. 1951, 293-297.

(34) Ohmura, K.; Thürlimann, C. M.; Kipf, M.; Carbajal, J. P.; Villez, K. Characterizing Long-Term Wear and Tear of lon-Selective PH Sensors. Water Sci. Technol. 2019, 80 (3), 541-550. https://doi.org/10.2166/wst.2019.301.

(35) Wald, A. Sequential Tests of Statistical Hypotheses. Ann. Math. Stat. 1945, 16 (2), 117-186. https://doi.org/10.1214/aoms/1177731118.

(36) Sedlak, D. Water 4.0: The Past, Present, and Future of the World's Most Vital Resource; Yale University Press: New Haven, CT, USA, 2014.

(37) Lotka, A. J. A Contribution to the Theory of Self-Renewing Aggregates, With Special Reference to Industrial Replacement. Ann. Math. Stat. 1939, 10 (1), 1-25.

https://doi.org/10.1214/aoms/1177732243.

(38) Baeriswyl, P.; Carisch, M.; Etter, B.; Holliger, U.; Hug, T.; Konz, N.; Lehmann, S.; Maron, T.; Oetterli, M.; Schneider, M. Y.; Tinner, B.; Udert, K. M. Abwasser im ländlichen Raum (wastewater in rural area, in German); VSA: Glattbrugg, 2017. 
(39) Levett, K. J.; Vanderzalm, J. L.; Page, D. W.; Dillon, P. J. Factors Affecting the Performance and Risks to Human Health of On-Site Wastewater Treatment Systems. Water Sci. Technol. 2010, 62 (7), 1499-1509. https://doi.org/10.2166/wst.2010.434.

(40) Ministry of the Environment. Johkasou Act (Tentative Translation); Act No. 43, Revised version Act No. 69; Japan, 1983.

(41) U.S. Environmental Protection Agency. Handbook for Managing Onsite and Clustered (Decentralized) Wastewater Treatment Systems. 2005, EPA-832-B-05-001.

(42) Government of South Australia. On-Site Wastewater Systems Code. April 2013.

(43) DIBt. Zulassungsgrundsätze Für Allgemeine Bauaufsichtliche Zulassungen Für Kleinkläranlagen (in German). 2012, 1-15.

(44) DWA. DWA-M 221 Grundsätze für Bemessung, Bau und Betrieb von Kleinkläranlagen mit aerober biologischer Reinigungsstufe (in German); Hennef, 2012.

(45) Schalk, T. Möglichkeiten zur Behandlung saisonal anfallender Abwässer in ländlich strukturierten Gebieten durch Anpassung der Bemessungsgrundlagen und temporäre Aktivierung interner Reserven (in German). Dresdener Berichte, Band 43, TU Dresden, Dresden, 2017.

(46) Mutzner, L.; Staufer, P.; Ort, C. Model-Based Screening for Critical Wet-Weather Discharges Related to Micropollutants from Urban Areas. Water Res. 2016, 104, 547-557. https://doi.org/10.1016/j.watres.2016.08.003.

(47) Rutsch, M.; Rieckermann, J.; Krebs, P. Quantification of Sewer Leakage: A Review. Water Sci. Technol. 2006, 54 (6-7), 135-144. https://doi.org/10.2166/wst.2006.616.

(48) Watanabe, R.; Harada, H.; Yasui, H.; Le, T. V.; Fujii, S. Exfiltration and Infiltration Effect on Sewage Flow and Quality: A Case Study of Hue, Vietnam. Environ. Technol. 2019, 1-11. https://doi.org/10.1080/09593330.2019.1680739.

(49) Graham, S.; Thrift, N. Out of Order: Understanding Repair and Maintenance. Theory Cult. Soc. 2007, 24 (3), 1-25. https://doi.org/10.1177/0263276407075954.

(50) Reymond, P.; Abdel Wahaab, R.; Moussa, M. S.; Lüthi, C. Scaling up Small Scale Wastewater Treatment Systems in Low- and Middle-Income Countries: An Analysis of Challenges and Ways Forward through the Case of Egypt. Util. Policy 2018, 52, 13-21. https://doi.org/10.1016/j.jup.2018.03.008. 